\title{
Toll-like receptor dependent autoantigens in animal models and humans in use to improve collagen induced arthritis
}

\author{
B Marklein ${ }^{2}$, Z Konthur ${ }^{1}$, T Häupl ${ }^{2}$, M J Shlomchik SR. ${ }^{3}$, G Steiner ${ }^{4}$, H Lehrach ${ }^{1}$, G R Burmester ${ }^{2}$, \\ F Apparailly ${ }^{5}$, K Skriner $^{2^{*}}$
}

From 5th European Workshop on Immune-Mediated Inflammatory Diseases

Sitges-Barcelona, Spain. 1-3 December 2010

\section{Objective}

This study was conducted with sera from patients with rheumatoid arthritis, systemic lupus erythematosus as well as with arthritis and lupus-like disease animal models to identify identical autoantigens in human and animal models for disease modifying use.

\section{Methods}

Using protein filter technology (28000 human protein filter) the autoantigen profile of RA and SLE patients, mouse collagen and zymosan induced arthritis, as well as collagen and pristan induced arthritis in rats and TLR7, TLR9 deficient double-deficient and MyoD88 and Tir8 deficient mice of the MRL-lpr/lpr background were obtained. Cationic liposomes transferring siRNAs were used for the validation of their potential as therapeutic target in collagen induced arthritis in mice (CIA).

\section{Results}

By high throughput methods using proteins filters, over 200 clones were identified to similarly bind patient, mouse and rat immunoglobulins. We could identify that up to $90 \%$ of all autoantigens including anti-histone, snRNP, hnRNP, t-RNA synthetases, PM, Scl2, Hsp proteins, and 17 different ribosomal autoantigens are MyoD88 dependent and influenced by Tir8. We found 18 identical proteins targeted in human and animal situations of arthritis. In contrast, autoantigens such as citrullinated proteins are generated independently of MyoD88 Toll7, 9 and Tir8. Systemic administration of
siRNAs in CIA mice with cationic liposomes inhibiting expression of Toll dependent autoantigens were used for the validation of their potential as therapeutic target in RA.

\section{Conclusion}

These novel data indicate that nucleic acid-binding proteins are targeted in animal models and patients with RA. Systemic blocking of common RNA or DNA binding proteins overexpessed in synovial target tissue appears to modify arthritis.

\section{Author details}

${ }^{1}$ Max-Planck-Institute for Molecular Genetics, Berlin, Germany. ${ }^{2}$ Charité University Medicine, Dept. of Rheumatology and Clinical Immunology, Humboldt University and Free University, Berlin, Germany. ${ }^{3}$ Dept. of Immunobiology, Yale University School of Medicine, New Haven, CT, USA. ${ }^{4}$ University Depts. of the Vienna Biocenter, Vienna, Austria. ${ }^{5}$ INSERM, U844, Hôpital Saint Eloi, and Université Montpellier I, Montpellier, France.

Published: 25 November 2010

doi:10.1186/1479-5876-8-S1-P53

Cite this article as: Marklein et al.: Toll-like receptor dependent autoantigens in animal models and humans in use to improve collagen induced arthritis. Journal of Translational Medicine 2010 8(Suppl 1):P53. 